$10.1117 / 2.1200610 .0445$

\title{
Fiber pressure sensors find application in urology
}

David Towers, Amanda McCartney, Michal Bialkowski, James Barton, Laurence Stewart, Catherine Towers, William MacPherson, and Robert Reuben

Silicone materials and broadband optical interrogation dramatically improve the sensitivity of small interferometric fiber pressure sensors of relevance to biomedicine.

Miniaturized sensors are of general interest for biomedical use, particularly to complement minimally invasive interventions where the entry point is along a body duct, such as the arterial system or urethra. However, probe sizes currently complicate and limit their applications. For example, conventional urological investigations monitor bladder pressure as a function of volume emitted during voiding, and require a pressure resolution less than $10 \mathrm{mbar}$. The pressure measurements are currently obtained via polymer tubes to external transducers or, less commonly, with miniature transducers. ${ }^{1}$ The probe size-between 2 and $3 \mathrm{~mm}$ diameter-affects patient physiology during bladder voiding and filling, and the possibility of using arrays for enhanced diagnostic information is very limited.

Such problems can be addressed with miniature fiber-opticbased pressure sensors that are compatible with single-point entry into the body. Typically, the fabrication of these instruments involves inserting a single-mode fiber of $125 \mu \mathrm{m}$ diameter cladding into a sensor body that is sealed by a diaphragm to give sensitivity to external pressure, as shown in Figure 1. The pressure is measured by tracking the effective cavity length as the diaphragm flexes. The cavity length itself is determined from light fringes (see Figure 2) formed by interference between reflections from the diaphragm and the fiber end. While this can be achieved using multiple narrowband lasers, ${ }^{2}$ the cost would be prohibitive for clinical use. Instead, in our approach, we employ a halogen bulb to provide a broadband source.

The sensor diaphragms were formed by spin-coating a thin, $10-50 \mu \mathrm{m}$ layer of silicone, in this case silicone certified for use in the body (Nusil MED10-6605). ${ }^{3}$ The material boosted sensitivity one to two orders of magnitude ${ }^{4}$ over that achieved in our earlier efforts with materials such as $\mathrm{Si}_{3} \mathrm{~N}_{4}$ and $\mathrm{Cu}$.

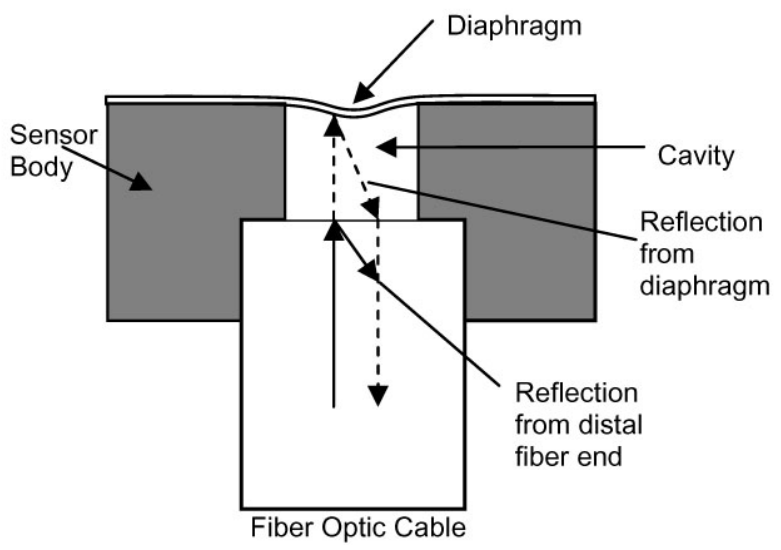

Figure 1. In this fiber-optic-based sensor, pressureflexes the diaphragm and changes the effective cavity length, which can be determined from interference between reflections from the fiber end and diaphragm.

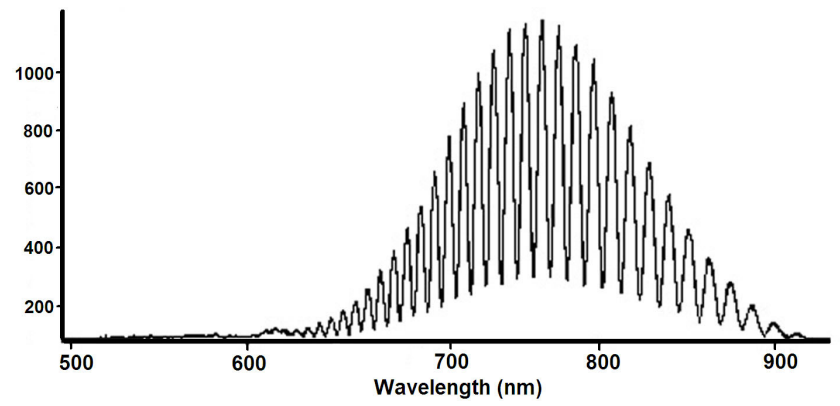

Figure 2. Shown is the intensity distribution (in arbitrary units) at a spectrometer from broadband illumination of a fiber pressure sensor. The nonuniform sinusoidal modulations, called fringes, are caused by the wavelength-dependent interference phase.

Two methods were used to make the silicon sensor bodies. In one, opposing holes, as shown in Figure 1, were etched for the fiber and diaphragm. Alternatively, single-mode fiber connector

Continued on next page 


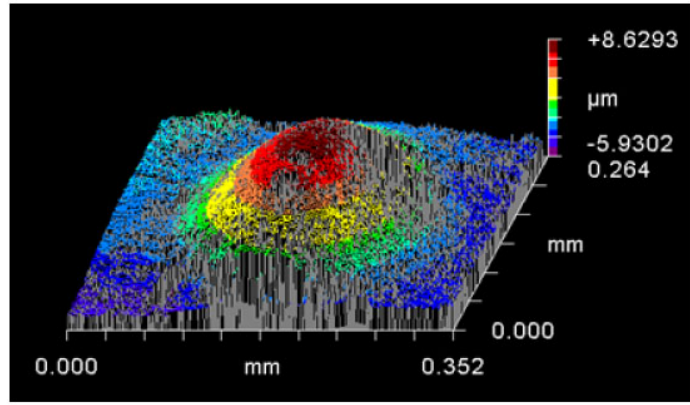

Figure 3. Shown is the deflection under internal pressure of a silicone diaphragm that has been bonded to the sensor body. The measurements were obtained using a white-light interferometer.

ferrules with a $126 \mu \mathrm{m}$ diameter through-hole were used. In both cases, the silicone layer was applied to the sensor body during the cure process. Prior to bonding the fiber into the sensor body, the diaphragm's deflection under pressure from within the cavity was characterized using a white light interferometer. Figure 3 shows a typical deformed profile.

The fringes obtained at the spectrometer were nonuniformly spaced because the phase change is inversely proportional to optical wavelength. The fringes were resampled at constant frequency intervals, and the Fourier transform of the result was calculated, yielding a well-defined power spectrum peak corresponding to the diaphragm distance. As the source bandwidth increases, more fringes develop, improving the measurement resolution. We have shown that a resolution of $\sim 2 \mathrm{~nm}$ is achievable with a 100nm bandwidth source.

Initial tests indicate that diaphragm thicknesses exceeding $40 \mu \mathrm{m}$ are needed in order to fabricate structurally reliable sensors that accommodate the heterogeneous silicone structure. Results from prototype sensors in a pressure-controlled chamber have confirmed a roughly linear increase of diaphragm deflection with pressure, as shown in Figure 4. We have shown that a resolution of $8 \mathrm{mbar}$ can be achieved that is largely limited by the stability of the experimental setup. ${ }^{4}$

The work to date has shown that fiber pressure sensors with silicone diaphragms and white light interferometric detection can provide the required measurement resolution at an acceptable system cost. The bandwidth of available spectrometers offers the potential to interrogate an array of 6-8 sensors. Future work will focus on fabrication issues in bonding the diaphragm to the sensor body. When these are resolved, further sensor miniaturization would be enabled, followed by initial patient trials.

The authors would like to thank the Engineering and Physical Sciences Research Council (EPSRC, UK) for funding under grant reference GR/S96555/01.

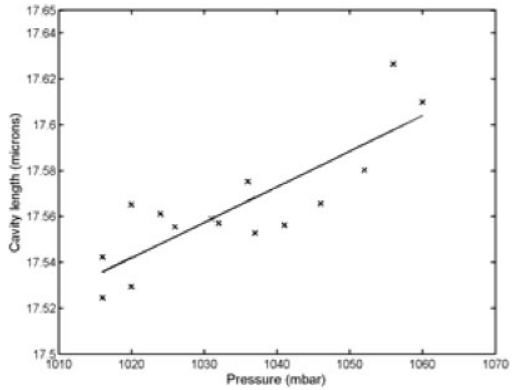

Figure 4. The sensor's diaphragm deflection ('cavity length') varies approximately linearly with the applied pressure.

\section{Author Information}

\section{David Towers and Catherine Towers}

School of Mechanical Engineering

University of Leeds

Leeds, UK

Dave Towers is a professor of optical instrumentation at the University of Leeds. His current research includes the application of optical sensors to live-cell imaging, particle-based diagnostics for volumetric flow measurement, and multi-constituent flows. His work includes sensor development for use in the urinary tract, as well as for multi-parameter sensing, for example, form, color, and surface texture.

Amanda McCartney, Michal Bialkowski, James Barton, William MacPherson, and Robert Reuben

School of Engineering and Physical Sciences

Heriot-Watt University

Edinburgh, UK

\section{Laurence Stewart}

Department of Urology

Western General Hospital

Edinburgh, UK

\section{References}

1. Y. Homma, J. Batista, et al., Urodynamics, in P. Abrams, L. Cardozo, S. Khoury, and A. Wein (eds.), Incontinence, $2^{\text {ed }}$ ed., 2001.

2. M. Gander, W. N. MacPherson, et al., Embedded micromachined fiber-optic FabryPerot sensors in aerodynamics applications, IEEE Sensors J. 3 (1), pp. 102-107, 2003. doi:10.1109/JSEN.2003.810099.

3. T. H. J. Yang, S. K. W. Leung, et al., In vitro dynamic micro-probing and the mechanical properties of human prostate tissues Regensberg Appl. Biomech., 2005.

4. A. J. McCartney, M. Bialkowski, et al., Interferometric sensors for application in the bladder and the lower urinary tract, Proc. SPIE 6293, 2006. doi:10.1117/12.681566. 